\title{
Partly occupied Wannier functions: Construction and applications
}

\author{
Thygesen, Kristian Sommer; Hansen, Lars Bruno; Jacobsen, Karsten Wedel
}

\section{Published in:}

Physical Review B Condensed Matter

Link to article, DOI:

10.1103/PhysRevB.72.125119

Publication date:

2005

Document Version

Publisher's PDF, also known as Version of record

Link back to DTU Orbit

\section{Citation (APA):}

Thygesen, K. S., Hansen, L. B., \& Jacobsen, K. W. (2005). Partly occupied Wannier functions: Construction and applications. Physical Review B Condensed Matter, 72(12), 125119.

https://doi.org/10.1103/PhysRevB.72.125119

\section{General rights}

Copyright and moral rights for the publications made accessible in the public portal are retained by the authors and/or other copyright owners and it is a condition of accessing publications that users recognise and abide by the legal requirements associated with these rights.

- Users may download and print one copy of any publication from the public portal for the purpose of private study or research.

- You may not further distribute the material or use it for any profit-making activity or commercial gain

- You may freely distribute the URL identifying the publication in the public portal 


\title{
Partly occupied Wannier functions: Construction and applications
}

\author{
K. S. Thygesen, L. B. Hansen, and K. W. Jacobsen \\ Center for Atomic-scale Materials Physics, Department of Physics, Technical University of Denmark, DK - 2800 Kgs. Lyngby, Denmark
}

(Received 14 June 2005; published 21 September 2005)

\begin{abstract}
We have developed a practical scheme to construct partly occupied, maximally localized Wannier functions (WFs) for a wide range of systems. We explain and demonstrate how the inclusion of selected unoccupied states in the definition of the WFs can improve both their localization and symmetry properties. A systematic selection of the relevant unoccupied states is achieved by minimizing the spread of the resulting WFs. The method is applied to a silicon cluster, a copper crystal, and a $\mathrm{Cu}(100)$ surface with nitrogen adsorbed. In all cases we demonstrate the existence of a set of WFs with particularly good localization and symmetry properties, and we show that this set of WFs is characterized by a maximal average localization.
\end{abstract}

DOI: 10.1103/PhysRevB.72.125119

PACS number(s): 71.15.Ap, 31.15.Ew, 31.15.Rh

\section{INTRODUCTION}

A characteristic property of the single-particle eigenstates of most molecular and solid state systems is their delocalized nature. For many practical purposes this property is undesired and the construction of equivalent representations in terms of localized orbitals becomes an important issue.

Within the independent-particle approximation the use of Wannier functions (WFs) allows for an exact description of the electronic ground state in terms of a minimal set of localized orbitals. ${ }^{1}$ The Wannier basis is truly minimal in the sense that the number of orbitals is just enough to accomodate the valence electrons of the system. Moreover, these localized WFs provide a formal justification of the widely used tight-binding ${ }^{2}$ and Hubbard models. ${ }^{3}$ Being the local analog of the extended Bloch states of solid state physics, the WFs formalize standard chemical concepts such as bonding, coordination, and electron lone pairs. Among the more technical applications of Wannier functions we mention the connection to polarization theory ${ }^{4,5}$ and their use within socalled "linear scaling" or "order- $N$ " methods to obtain the electronic groundstate. ${ }^{6}$ Very recently numerical methods for electron transport calculations employing a Wannier function basis set have been developed. ${ }^{7,8}$

In the context of molecular systems the analog of Wannier functions for finite systems has been studied under the name "localized molecular orbitals."9-14 These are traditionally defined by an appropriate unitary transformation of the occupied single-particle eigenstates and have been used for investigation of chemical bonding. In the following we shall for simplicity use the term WF to cover also localized molecular orbitals.

In 1997, Marzari and Vanderbilt developed a scheme to perform practical calculations of maximally localized Wannier functions for an isolated group of bands, i.e., a set of bands which is separated by a finite gap from all higher- and lower-lying bands. ${ }^{15}$ Within this scheme, the usual arbitrariness inherent in the definition of the Wannier functions due to the unspecified set of unitary transformations of the Bloch states at every wave vector, is removed by requiring that the sum of second moments of the resulting WFs is minimal. The method follows the traditional idea of defining Wannier functions by a unitary transformation of the occupied
(Bloch) orbitals. In general, such methods fail to produce well-localized orbitals when applied to metallic systems because the unoccupied states belonging to the partly filled valence bands ${ }^{16}$ are not considered. Of course, in cases where the partly filled valence bands are separated by a gap from all higher bands, the method of Marzari and Vanderbilt still applies. However, in the more general case where the bands of interest cross and/or hybridize with other unwanted bands a different approach must be used.

In this paper we demonstrate how the localization and in some cases also the symmetry of a set of WFs can be drastically improved by including selected unoccupied states in the definition of the WFs. ${ }^{17}$ The determination of the relevant unoccupied states can be viewed as a bonding-antibonding closing procedure, where occupied bonding states are paired with their antibonding counterparts to yield localized orbitals. To be more specific, consider two well-localized atomic orbitals on neighboring atoms in a molecule. If we allow the two states to hybridize, a bonding and an antibonding combination will result-combinations which may be less localized than the individual atomic orbitals. To regain the localized atomic orbitals from the molecular orbitals we need both the bonding and antibonding combination independent of their occupation, see Fig. 1. In some cases the antibonding state may have hybridized further with other states and the state which "matches" the bonding state will be a linear combination of eigenstates. The problem we address here is the construction of a method for systematically identifying the relevant unoccupied states. We show that this can be achieved by optimizing the localization of the resulting WFs.

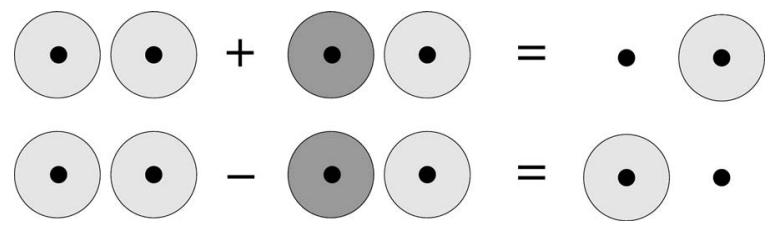

FIG. 1. Schematic of the bonding-antibonding closure for a hydrogen molecule. The construction of well-localized atomic $s$ orbitals involves a matching of bonding and antibonding orbitals, independent of their occupation. The sign of the wave functions is indicated by the shading. 
The paper gives a more detailed and extended account of the work previously published in a paper ${ }^{17}$

For periodic systems the bonding-antibonding closure can be viewed as a procedure for disentangling the partly occupied valence bands from higher-lying bands. This problem has previously been addressed by Souza et al. ${ }^{18}$ who proposed a disentangling method based on a minimization of the change in character of the Bloch states across the Brillouin zone (BZ). While this is a natural strategy for crystalline systems, it is not clear how this disentanglement procedure applies to nonperiodic systems like isolated molecules, a surface with adsorbates, or a metal with impurities.

The present method is related to that of Souza et al., ${ }^{18}$ however, instead of minimizing the dispersion across the BZ we suggest a disentanglement procedure based exclusively on a minimization of the spread of the WFs. In this way we omit any reference to the wave vector and are therefore not limited to periodic systems. The generality of the method is demonstrated by application to three different systems: an isolated $\mathrm{Si}_{5}$ cluster, a copper crystal, and a $\mathrm{Cu}(100)$ surface with nitrogen adsorbed. Our results for the copper crystal are very similar to those obtained by Souza et al., ${ }^{18}$ and this indicates the similarity of the two localization schemes for periodic systems.

The paper is organized as follows: In Sec. II we introduce the spread functional and outline the strategy behind the localization algorithm. In Sec. III we give the formal definition of partly occupied WFs in the limiting case of a large supercell and derive the corresponding expressions for the gradient of the spread functional. The extension to periodic systems is discussed in Sec. IV. In Sec. V we apply the method to a $\mathrm{Si}_{5}$ cluster, a copper crystal, and a $\mathrm{Cu}(100)$ surface with adsorbed nitrogen.

\section{DESCRIPTION OF THE METHOD}

In this section we introduce the spread functional used to measure the degree of localization of a set of orbitals, and give an introductory description of the localization scheme including its relation to the method of Souza et al. ${ }^{18}$

\section{A. Spread functional}

Within the localization scheme of Marzari and Vanderbilt ${ }^{15}$ the spread of a set of functions $\left\{w_{n}(\mathbf{r})\right\}_{n=1}^{N}$ is measured by the sum of second moments

$$
S=\sum_{n=1}^{N}\left(\left\langle w_{n}\left|r^{2}\right| w_{n}\right\rangle-\left\langle w_{n}|\mathbf{r}| w_{n}\right\rangle^{2}\right) .
$$

When periodic boundary conditions are applied, as in the present study, and the supercell is sufficiently large, the minimization of $S$ is equivalent to the maximization of ${ }^{19}$

$$
\Omega=\sum_{n=1}^{N} \sum_{\alpha=1}^{N_{G}} W_{\alpha}\left|Z_{\alpha, n n}\right|^{2},
$$

where the matrix $Z_{\alpha}$ is defined as

$$
Z_{\alpha, n m}=\left\langle w_{n}\left|e^{-i \mathbf{G}_{\alpha} \cdot \mathbf{r}}\right| w_{m}\right\rangle .
$$

The $\left\{\mathbf{G}_{\alpha}\right\}$ is a set of at most six reciprocal lattice vectors and $\left\{W_{\alpha}\right\}$ are corresponding weights which account for the shape of the unit cell. For a definition and discussion of these quantities we refer to Refs. 13 and 14.

\section{B. Localization scheme}

The starting point is the set of single-particle eigenstates $\left\{\psi_{n}\right\}$ resulting from a conventional electronic structure calculation. For simplicity we shall assume that the system is isolated or is contained in a large supercell such that reference to $\mathbf{k}$ points can be omitted. The aim is to obtain a set of $N_{w}$ localized WFs with the property that any eigenstate below a specified energy $E_{0}$ can be exactly reproduced as a linear combination of the WFs. An obvious way to achieve this would be to apply the method of Marzari and Vanderbilt to compute the unitary transformation of the $N_{w}$ lowest eigenstates leading to the most localized WFs. The problem with this strategy is, however, that it is in general not possible to localize all WFs simultaneously, and the problem cannot be overcome by increasing $N_{w}$.

Instead, we define an external localization space as the space spanned by the $N_{b}$ lowest-lying eigenstates $\left(N_{b}>N_{w}\right)$. Within this space we consider the subspace spanned by the eigenstates with energy below $E_{0}$, together with $L$ extra degrees of freedom (EDF). We shall refer to this subspace as the active localization space or simply the localization space. The EDF are assumed to be orthogonal and $L$ is chosen such that the dimension of the active localization space equals $N_{w}$. We then perform a simultaneous optimization of the WFs within the active localization space and of the active localization space itself. In practice this is achieved by optimizing an $N_{w} \times N_{w}$ unitary matrix together with the coordinates of the EDF such that the functional $\Omega$ becomes maximal.

It is the determination of the EDF that distinguishes our method from that of Souza et al. ${ }^{18}$ In the latter, the spread functional is decomposed into two terms: $\Omega=\Omega_{I}+\widetilde{\Omega}$, where $\Omega_{I}$ is related to the $k$-space dispersion of the band-projection operator, see Ref. 18. In the first step, the EDF are determined by maximizing $\Omega_{l}$, which depends only on the localization space itself and not on the internal unitary transformation. In the second step $\widetilde{\Omega}$, or equivalently $\Omega$, is then maximized within the fixed localization space. It is clear that the separate maximization of $\Omega_{I}$ and $\widetilde{\Omega}$ does not amount to the global maximization of $\Omega$ that we propose here. We shall, however, see that the two methods lead to very similar results in the case of periodic systems.

\section{LARGE SUPERCELLS}

In this section we give a detailed description of the localization scheme in the limiting case of a large supercell where a $\Gamma$-point sampling of the first Brillouin zone is a good approximation. For simplicity we discuss this case separately before extending it to periodic systems, although the latter contains the former as a special case. After giving the definition of partly occupied Wannier functions we derive ex- 
pressions for the gradients of the spread functional and discuss how to combine these with a Lagrange multiplier scheme to determine the maximum of $\Omega$.

\section{A. Definition of partly occupied Wannier functions}

We denote the total number of eigenstates obtained from the electronic structure calculation by $N_{b}$ and the number of eigenstates below the energy $E_{0}$ by $M$. Our aim is to construct a set of $N_{w}$ WFs which span at least the $M$ lowest-lying eigenstates. The remaining $L=N_{w}-M$ degrees of freedom are simply used to improve the localization of the resulting WFs as much as possible. We expand the WFs in terms of the $M$ lowest-lying eigenstates and $L$ extra degrees of freedom $\left\{\phi_{l}\right\}$ belonging to the $\left(N_{b}-M\right)$-dimensional space of eigenstates with energy above $E_{0}$

$$
w_{n}=\sum_{m=1}^{M} U_{m n} \psi_{m}+\sum_{l=1}^{L} U_{M+l, n} \phi_{l},
$$

where the extra degrees of freedom (EDF) are written as

$$
\phi_{l}=\sum_{m=1}^{N_{b}-M} c_{m l} \psi_{M+m} .
$$

The columns of the matrix $c$ are orthonormal and represent the coordinates of the EDF with respect to the eigenstates lying above $E_{0}$. The matrix $U$ is unitary and represents a rotation of the functions $\left\{\psi_{1}, \ldots, \psi_{M}, \phi_{1}, \ldots, \phi_{L}\right\}$.

In order to simplify the notation we introduce the matrices

$$
C=\left[\begin{array}{cc}
I^{M \times M} & 0 \\
0 & c
\end{array}\right], \quad V=C U=\left[\begin{array}{c}
U^{M} \\
c U_{L}
\end{array}\right],
$$

where $U^{M}$ and $U_{L}$ denotes the $M$ upper-most and $L$ lowermost rows of $U$, respectively. The $i$ th column of $V$ gives the coordinates of $w_{i}$ with respect to the full set of eigenstates $\left\{\psi_{n}\right\}$.

Substituting the expansions (4) and (5) into Eq. (3) we obtain a compact matrix expression

$$
Z_{\alpha}=V^{\dagger} Z_{\alpha}^{(0)} V=U^{\dagger} C^{\dagger} Z_{\alpha}^{(0)} C U,
$$

where $Z_{\alpha}^{(0)}$ is obtained from Eq. (3) by using the eigenstates $\left\{\psi_{n}\right\}$ in the inner product,

$$
Z_{\alpha, n m}^{(0)}=\left\langle\psi_{n}\left|e^{-i \mathbf{G}_{\alpha} \cdot \mathbf{r}}\right| \psi_{m}\right\rangle .
$$

\section{B. Gradient of $\Omega$}

Through Eq. (7) the spread functional $\Omega$ in Eq. (2) becomes a function of the matrices $U$ and $c$. The maximum of $\Omega$ can be found iteratively by updating $U$ and $c$ in the direction given by the gradient. In the following we derive expressions for the gradient of $\Omega$.

We write the unitary matrix at iteration $n$ as $U^{(n)}$ $=U^{(n-1)} \exp (-A)$, where $A$ is an anti-Hermitian matrix. Since we are only concerned with small variations, we expand the exponential to first order, i.e., $\exp (-A) \simeq 1-A$. Inserting this into Eqs. (2) and (7) we find

$$
\frac{\partial \Omega}{\partial A_{i j}}=\sum_{\alpha=1}^{N_{G}} W_{\alpha}\left[Z_{\alpha, j i}\left(Z_{\alpha, j j}^{*}-Z_{\alpha, i i}^{*}\right)-Z_{\alpha, i j}^{*}\left(Z_{\alpha, i i}-Z_{\alpha, j j}\right)\right] .
$$

All matrices in this expression refer to iteration $n-1$. The new rotation at iteration $n$ is then obtained by multiplying $U^{(n-1)}$ by $\exp \left[-d\left(\nabla_{A} \Omega\right)\right]$ where $d$ is the length of the steepest-ascent step and $\left[\nabla_{A} \Omega\right]_{i j}=\partial \Omega / \partial A_{i j}$.

We now turn to the problem of determining the steepest uphill direction of $\Omega$ with respect to variations in $c$. In general, for a real-valued function $f(z=x+i y)$ the direction of steepest ascent with respect to $z$ is given by

$$
\frac{\partial f}{\partial z^{*}} \equiv \frac{1}{2}\left(\frac{\partial f}{\partial x}+i \frac{\partial f}{\partial y}\right)
$$

To calculate the gradient $\partial \Omega / \partial c_{i j}^{*}$ we use that

$$
\frac{\partial\left|Z_{\alpha, n n}\right|^{2}}{\partial c_{i j}^{*}}=Z_{\alpha, n n} \frac{\partial Z_{\alpha, n n}^{*}}{\partial c_{i j}^{*}}+Z_{\alpha, n n}^{*} \frac{\partial Z_{\alpha, n n}}{\partial c_{i j}^{*}} .
$$

From Eq. (7) it follows that

$$
\begin{aligned}
\frac{\partial Z_{\alpha, n n}}{\partial c_{i j}^{*}}= & \sum_{a b c d} U_{n a}^{\dagger} \frac{\partial C_{a b}^{\dagger}}{\partial c_{i j}^{*}} Z_{\alpha, b c}^{(0)} C_{c d} U_{d n} \\
& +\sum_{a b c d} U_{n a}^{\dagger} C_{a b}^{\dagger} Z_{\alpha, b c}^{(0)} \frac{\partial C_{c d}}{\partial c_{i j}^{*}} U_{d n},
\end{aligned}
$$

and from definition (6)

$$
\begin{gathered}
\frac{\partial C_{n m}}{\partial c_{i j}^{*}}=0 \\
\frac{\partial C_{n m}^{\dagger}}{\partial c_{i j}^{*}}=\delta_{m, M+i} \delta_{n, M+j} .
\end{gathered}
$$

It is now easy to establish that

$$
\frac{\partial Z_{\alpha, n n}}{\partial c_{i j}^{*}}=\left[Z_{\alpha}^{(0)} V\right]_{M+i, n} U_{M+j, n}^{*}
$$

$$
\frac{\partial Z_{\alpha, n n}^{*}}{\partial c_{i j}^{*}}=\left[\left(Z_{\alpha}^{(0)}\right)^{\dagger} V\right]_{M+i, n} U_{M+j, n}^{*} .
$$

Combining Eq. (11) with Eqs. (15) and (16) we arrive at the desired expression

$$
\frac{\partial \Omega}{\partial c_{i j}^{*}}=\sum_{\alpha=1}^{N_{G}} W_{\alpha}\left[Z_{\alpha}^{(0)} V D\left(Z_{\alpha}^{*}\right) U^{\dagger}+\left(Z_{\alpha}^{(0)}\right)^{\dagger} V D\left(Z_{\alpha}\right) U^{\dagger}\right]_{M+i, M+j},
$$

where $D\left(Z_{\alpha}\right)$ is a diagonal matrix with $\left(Z_{\alpha, n n}\right)$ in the diagonal.

To treat the constraint that the EDF $\left\{\phi_{l}\right\}$ should be orthonormal during the maximization procedure we introduce the Lagrange multipliers $\lambda_{i j}$ and perform an unconstrained maximization of the functional 


$$
\Omega_{L}=\Omega-\sum_{i j} \lambda_{i j}\left\langle\phi_{i} \mid \phi_{j}\right\rangle .
$$

The Lagrange multipliers are initially unknown and must be estimated at each iteration. At the maximum we have $\nabla_{c^{*}} \Omega_{L}=0$ which is equivalent to the condition

$$
\nabla_{c} c^{*}-c \lambda^{\mathrm{T}}=0 .
$$

Multiplying by $c^{\dagger}$ from the left leads to

$$
\lambda^{\mathrm{T}}=c^{\dagger} \nabla_{c^{*} \Omega} .
$$

This relation can be used to estimate the Lagrange multipliers at each iteration. A step of length $d$ in the steepest uphill direction is thus accomplished by adding to $c$ the matrix $d\left(1-c c^{\dagger}\right) \nabla_{c^{*}} \Omega$, followed by an orthonormalization of the columns of $c$.

\section{PERIODIC SYSTEMS}

We consider a periodic system with a unit cell defined by basis vectors $\mathbf{a}_{1}, \mathbf{a}_{2}, \mathbf{a}_{3}$ which in turn define the basis vectors of the reciprocal lattice $\mathbf{b}_{1}, \mathbf{b}_{2}, \mathbf{b}_{3}$. The Bloch states, $\left\{\psi_{n \mathbf{k}}\right\}$, resulting from the electronic structure calculation are characterized by a band index $n$ and a crystal momentum $\mathbf{k}$. The total number of bands is denoted by $N_{b}$ and the number of eigenstates at a given $\mathbf{k}$ point with energy below $E_{0}$ is denoted by $M_{\mathbf{k}}$. We assume a uniform sampling of the first BZ such that any $\mathbf{k}$ point can be written as

$$
\mathbf{k}=\frac{n_{1}}{N_{1}} \mathbf{b}_{1}+\frac{n_{2}}{N_{2}} \mathbf{b}_{2}+\frac{n_{3}}{N_{3}} \mathbf{b}_{3},
$$

where $N_{i}$ is the number of $\mathbf{k}$ points in the direction $\mathbf{b}_{i}$ and $n_{i}=0, \ldots, N_{i}-1$. Note that the $\Gamma$ point is always included. With this convention the Bloch states, $\left\{\psi_{n \mathbf{k}}\right\}$ correspond exactly to the $\Gamma$-point eigenstates of the repeated cell defined by the extended basis vectors $N_{1} \mathbf{a}_{1}, N_{2} \mathbf{a}_{2}, N_{3} \mathbf{a}_{3}$. An alternative way of stating this correspondence is to say that the $\mathbf{k}$ points in Eq. (21) fall on the reciprocal lattice of the repeated cell, see Fig. 2. As we shall see below, this correspondence allows us to use the spread functional $\Omega$ defined in Eq. (2) also for the periodic system. We stress that the formalism developed in the following section contains the $\Gamma$-point formalism described in the preceding sections as a special case.

\section{A. Definition of partly occupied Wannier functions}

We write the $n$th Wannier function related to unit cell $i$ as

$$
w_{i, n}=\frac{1}{\sqrt{N_{k}}} \sum_{\mathbf{k}} e^{-i \mathbf{k} \cdot \mathbf{R}_{i}} \widetilde{\psi}_{n \mathbf{k}}
$$

where $N_{k}$ is the total number of $\mathbf{k}$ points and $\widetilde{\psi}_{n \mathbf{k}}$ is a generalized Bloch state to be defined below. ${ }^{15}$ Each generalized band, i.e., each set $\left\{\tilde{\psi}_{n \mathbf{k}}\right\}$ for fixed $n$, gives rise to one WF per unit cell. These WFs are simply related by translation, i.e., $w_{i, n}(\mathbf{r})=w_{0, n}\left(\mathbf{r}-\mathbf{R}_{i}\right)$, and thus it suffices to consider the WFs of the cell at the origin. In doing this we can omit the cell

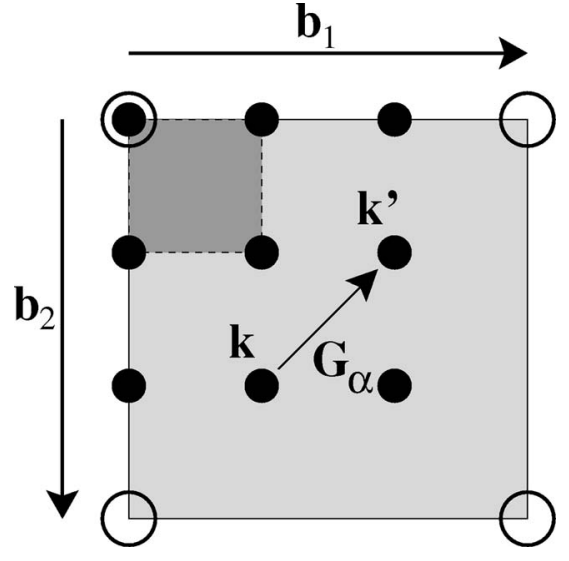

FIG. 2. Relation between the first BZ of the unit cell, defined by the reciprocal basis vectors $\mathbf{b}_{1}, \mathbf{b}_{2}, \mathbf{b}_{3}$ (light gray), and the first BZ of the repeated unit cell (dark gray). In this case $N_{1}$ and $N_{2}$ from Eq. (21) both equals 3. The relation between $\mathbf{k}$ and $\mathbf{k}^{\prime}$, given in Eq. (29), is indicated.

index and simply denote the WFs by $\left\{w_{n}\right\}$. We denote the number of WFs per cell by $N_{w}$.

Following the idea behind Eq. (4) we expand the generalized Bloch state $\widetilde{\psi}_{n \mathbf{k}}$ in terms of the $M_{\mathbf{k}}$ lowest-lying Bloch states and $L_{\mathbf{k}}$ extra degrees of freedom, $\left\{\phi_{l \mathbf{k}}\right\}$, from the remaining $\left(N_{b}-M_{\mathbf{k}}\right)$-dimensional space

$$
\tilde{\psi}_{n \mathbf{k}}=\sum_{m=1}^{M_{\mathbf{k}}} U_{m n}^{\mathbf{k}} \psi_{m \mathbf{k}}+\sum_{l=1}^{L_{\mathbf{k}}} U_{M_{\mathbf{k}}+l, n}^{\mathbf{k}} \phi_{l \mathbf{k}}
$$

where the EDF are expanded as

$$
\phi_{l \mathbf{k}}=\sum_{m=1}^{N_{b}-M_{\mathbf{k}}} c_{m l}^{\mathbf{k}} \psi_{M_{\mathbf{k}}+m, \mathbf{k}} .
$$

The number of EDF at a given $\mathbf{k}$ point is determined by the condition $L_{\mathbf{k}}+M_{\mathbf{k}}=N_{w}$. If $M_{\mathbf{k}}$ exceeds $N_{w}$, we simply put $M_{\mathbf{k}}=N_{w}$. Due to the exact correspondence between the Bloch states $\left\{\psi_{n \mathbf{k}}\right\}$ and the $\Gamma$-point eigenstates of the repeated cell, we can use the functional (2) to measure the spread of the Wannier functions. The matrices $Z_{\alpha}$ are still defined by Eq. (3) but it should be remembered that the inner product as well as the reciprocal lattice vector $\mathbf{G}_{\alpha}$ now refer to the repeated cell. From Eqs. (23) and (24) we find the following generalization of Eq. (7):

$$
Z_{\alpha}=\sum_{\mathbf{k}, \mathbf{k}^{\prime}} Z_{\alpha}^{\mathbf{k} \mathbf{k}^{\prime}}
$$

where

$$
Z_{\alpha}^{\mathbf{k} \mathbf{k}^{\prime}}=\left(U^{\mathbf{k}}\right)^{\dagger}\left(C^{\mathbf{k}}\right)^{\dagger} Z_{\alpha}^{(0), \mathbf{k} \mathbf{k}^{\prime}} C^{\mathbf{k}^{\prime}} U^{\mathbf{k}^{\prime}} .
$$

The matrix $C^{\mathbf{k}}$ is given by the obvious k-point analog of Eq. (6) and the matrix $Z_{\alpha}^{(0), \mathbf{k k}^{\prime}}$ is defined by 


$$
Z_{\alpha, n m}^{(0), \mathbf{k k}^{\prime}}=\left\langle\psi_{n \mathbf{k}}\left|e^{-i \mathbf{G}_{\alpha} \cdot \mathbf{r}}\right| \psi_{m \mathbf{k}^{\prime}}\right\rangle
$$

Most of the matrices $Z_{\alpha}^{(0), \mathbf{k k}^{\prime}}$ are in fact zero. Writing the Bloch functions as $\psi_{n \mathbf{k}}=u_{n \mathbf{k}}(\mathbf{r}) \exp (i \mathbf{k} \cdot \mathbf{r})$, where $u_{n \mathbf{k}}$ has the periodicity of the lattice, we get

$$
Z_{\alpha, n m}^{(0), \mathbf{k k}^{\prime}}=\int u_{n \mathbf{k}}^{*}(\mathbf{r}) u_{m \mathbf{k}^{\prime}}(\mathbf{r}) e^{i\left(\mathbf{k}^{\prime}-\mathbf{k}-\mathbf{G}_{\alpha}\right) \cdot \mathbf{r}} \mathrm{d} \mathbf{r}
$$

which is nonzero only when

$$
\mathbf{k}^{\prime}=\mathbf{k}+\mathbf{G}_{\alpha} .
$$

Here it is implicit that $\mathbf{k}$ and $\mathbf{k}^{\prime}$ belong to the first $\mathrm{BZ}$ and thus it might be necessary to translate $\mathbf{k}^{\prime}$ by a reciprocal lattice vector. The relation between $\mathbf{k}$ and $\mathbf{k}^{\prime}$ is illustrated in Fig. 2. Note that the condition in Eq. (29) reduces the double sum in Eq. (25) to a single sum over $\mathbf{k}$.

The derivation of the gradient of $\Omega$ follows closely the $\Gamma$-point case discussed in Sec. III B and is therefore omitted. The result is

$$
\begin{aligned}
& \frac{\partial \Omega}{\partial A_{i j}^{\mathbf{k}}}=\sum_{\alpha=1}^{N_{G}} W_{\alpha}\left[\left(Z_{\alpha, j j}\right)^{*} Z_{\alpha, j i}^{\mathbf{k}-\mathbf{G}_{\alpha}, \mathbf{k}}+Z_{\alpha, j j}\left(Z_{\alpha, i j}^{\left.\mathbf{k}, \mathbf{k}+\mathbf{G}_{\alpha}\right)^{*}}\right.\right. \\
& \left.-\left(Z_{\alpha, i i}\right)^{*} Z_{\alpha, j i}^{\mathbf{k}, \mathbf{k}+\mathbf{G}_{\alpha}}-Z_{\alpha, i i}\left(Z_{\alpha, i j}^{\mathbf{k}-\mathbf{G}_{\alpha}, \mathbf{k}}\right)^{*}\right] . \\
& \frac{\partial \Omega}{\partial\left(c_{i j}^{\mathbf{k}}\right)^{*}}=\sum_{\alpha=1}^{N_{G}} W_{\alpha}\left[Z_{\alpha}^{(0), \mathbf{k}, \mathbf{k}+\mathbf{G}_{\alpha}} V^{\mathbf{k}+\mathbf{G}_{\alpha}} D\left(Z_{\alpha}^{*}\right)\left(U^{\mathbf{k}}\right)^{\dagger}\right. \\
& \left.+\left(Z_{\alpha}^{(0), \mathbf{k}-\mathbf{G}_{\alpha}, \mathbf{k}}\right)^{\dagger} V^{\mathbf{k}-\mathbf{G}_{\alpha}} D\left(Z_{\alpha}\right)\left(U^{\mathbf{k}}\right)^{\dagger}\right]_{M_{\mathbf{k}}+i, M_{\mathbf{k}}+j}
\end{aligned}
$$

We note that these expressions, of course, reduce to Eqs. (9) and (17) in the limit of a single $\mathbf{k}$ point. The maximization of $\Omega$ proceeds along the same lines as for the $\Gamma$-point case, except that Lagrange multipliers are needed for each $\mathbf{k}$ point. For example the analog of Eq. (18) reads

$$
\Omega_{L}=\Omega-\sum_{i j, \mathbf{k}} \lambda_{i j, \mathbf{k}}\left\langle\phi_{i \mathbf{k}} \mid \phi_{j \mathbf{k}}\right\rangle
$$

\section{B. Optimizing the number of extra degrees of freedom}

For given values of $N_{b}, N_{w}$, and $E_{0}$, the algorithm introduced above produces the $N_{w}$ most localized WFs that can be formed within the external localization space when all eigenstates below $E_{0}$ should be exactly reproducible in terms of the WFs. It remains to determine the optimal values for $N_{b}$ and $N_{w}$ for a given $E_{0}$. Let us start by considering the situation where $N_{b}$ has been fixed at a value which is large enough to include all antibonding states relevant for the localization. In practice this typically means $\sim 10 \mathrm{eV}$ above the Fermi level. It seems as a natural strategy to choose $N_{w}$ such that the localization per orbital is maximal. To quantify this condition we define the average localization per orbital as

$$
\langle\Omega\rangle=\frac{\Omega\left[E_{0}, N_{b}, N_{w}\right]}{N_{w}},
$$

where we have indicated the dependence of $\Omega$ on the three parameters explicitly. We note that since the value of $\Omega$ also depends on the size and shape of the supercell, it does not make sense to compare the value of $\Omega$ for systems described in different supercells. Fixing $N_{w}$ on the basis of $\langle\Omega\rangle$ represents a completely general criterion which can be applied in any situation. However, the localization procedure must be carried out for several values of $N_{w}$ which might be a tedious task depending on the size of the system. We next consider the situation when $N_{b}$ is also allowed to change. Formally, the global maximum of $\langle\Omega\rangle$ is attained in the limit where both $N_{b}$ and $N_{w}$ tend to infinity in which case an infinite set of completely localized delta functions can be realized. However, we have found that for practical values of $N_{b}$ where very high energy states are not included in the external localization space, $\langle\Omega\rangle$ will have a local maximum for some $N_{w}$, and the position of the maximum is not sensitive to the actual value of $N_{b}$. Thus, it is indeed possible to determine an optimal value of $N_{w}$ by maximizing $\langle\Omega\rangle$.

Alternatively it is often possible to determine a value for $N_{w}$ based on symmetry arguments, chemical intuition, or a closed band condition. As we shall see in the following examples the two criteria for determining $N_{w}$ lead to similar results.

\section{Start guess for $U^{\mathrm{k}}$ and $c^{\mathrm{k}}$}

For small systems we have found that the localization algorithm is quite stable and usually leads to the global maximum independently of the initial value of the matrices $\left\{U^{\mathbf{k}}\right\},\left\{c^{\mathbf{k}}\right\}$. For larger systems, however, there is a risk of getting stuck in a local maximum and in such cases the start guess becomes important. It is then natural to start from a set of simple orbitals located either at the atoms or at the bond centers. Let $\left\{f_{\nu}\right\}$ denote such a set of simple orbitals. The question is how to transform this into the matrices $\left\{U^{\mathbf{k}}\right\},\left\{c^{\mathbf{k}}\right\}$. To this end we project the initial orbitals onto the subspace spanned by the Bloch states at each $\mathbf{k}$ point

$$
\tilde{f}_{\nu \mathbf{k}}=\sum_{n=1}^{N_{b}}\left\langle\psi_{n \mathbf{k}} \mid f_{\nu}\right\rangle \psi_{n \mathbf{k}}
$$

The following procedure is carried out for each $\mathbf{k}$ point separately. For fixed $\mathbf{k}$ we regard $\left\langle\psi_{n \mathbf{k}} \mid f_{\nu}\right\rangle$ as a matrix in the indices $n, \nu$. Its columns represent the coordinates of the $\widetilde{f}_{\mathbf{k} \nu}$ with respect to the Bloch states $\left\{\psi_{n \mathbf{k}}\right\}_{n=1}^{N_{b}}$ and as such it is a (nonorthogonalized) version of the matrix $V^{\mathbf{k}}$, see Eq. (6). After a normalization of the columns of $\left\langle\psi_{n \mathbf{k}} \mid f_{\nu}\right\rangle$ we compute the norm of the component of $\tilde{f}_{\nu \mathbf{k}}$ orthogonal to the occupied subspace

$$
\left\|\tilde{f}_{\nu \mathbf{k}}^{\perp}\right\|^{2}=\sum_{n=M(\mathbf{k})}^{N_{b}}\left|\left\langle\psi_{n \mathbf{k}} \mid f_{\nu}\right\rangle\right|^{2} .
$$

The first EDF is chosen as a normalized version of the $\tilde{f}_{\mathbf{k} \nu}^{\perp}$ for which $\left\|\tilde{f}_{\mathbf{k} \nu}^{\perp}\right\|$ is the largest. The remaining $\tilde{f}_{\nu}^{\perp}$ 's are then or- 
(a)

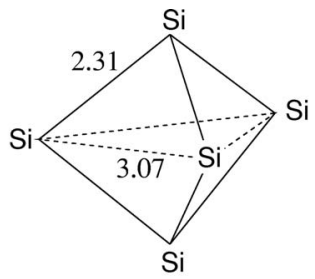

(b)

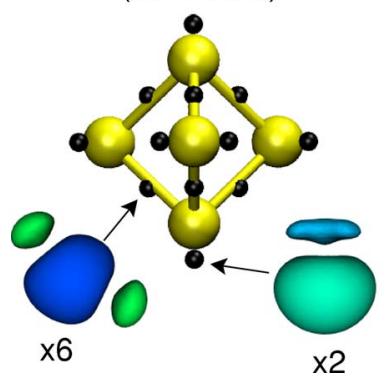

(Top view)

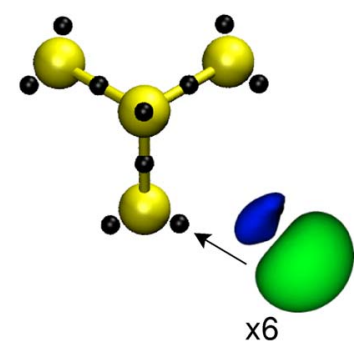

FIG. 3. (Color online) (a) Geometry of the $\mathrm{Si}_{5}$ cluster. (b) Contour plots of the WFs corresponding to $L=4$. The position of the WF centers are indicated by black spheres.

thogonalized onto this vector and the process is repeated until all EDF, and thus $c^{\mathbf{k}}$, have been determined. Finally the identity $U^{\mathbf{k}}=\left(C^{\mathbf{k}}\right)^{\dagger} V^{\mathbf{k}}$ with $V^{\mathbf{k}} \rightarrow\left\langle\psi_{n \mathbf{k}} \mid f_{\nu}\right\rangle$ determines $U^{\mathbf{k}}$. Since the $\widetilde{f}_{\nu \mathbf{k}}$ are not necessarily orthogonal, the columns of the resulting $U^{\mathbf{k}}$ must be explicitly orthogonalized.

\section{RESULTS}

In the following sections we apply the localization scheme to three different systems. To demonstrate the generality of the method we consider both isolated and metallic systems as well as a metal surface with adsorbed impurities. In Sec. V A we construct partly occupied WFs for an isolated $\mathrm{Si}_{5}$ cluster and illustrate how different sets of WFs can be obtained by varying the number of extra degrees of freedom. In Sec. V B we investigate the WFs of a $\mathrm{Cu}(\mathrm{fcc})$ crystal and compare the results with those obtained by Souza and coworkers ${ }^{18}$ who studied the same system using a different but related method. Finally, in Sec. V C we perform a detailed WF analysis for a $\mathrm{Cu}(100)$ surface with 0.5 monolayers of nitrogen. In all calculations we use a plane-wave based density-functional theory (DFT) $\operatorname{code}^{20}$ to obtain the KohnSham eigenstates, and we describe the ion potential by Vanderbilt ultrasoft pseudopotentials. ${ }^{21}$ To ensure a proper convergence of the unoccupied states employed in the localization scheme, the DFT calculations have been converged with respect to the full set of Kohn-Sham eigenvalues. In the Appendix we explain how to extend the localization scheme to ultrasoft pseudopotentials.

\section{A. $\mathrm{Si}_{5}$ cluster}

As an example of an isolated system we consider an $\mathrm{Si}_{5}$ cluster in its ground-state geometry, ${ }^{22}$ see Fig. 3(a). We use a cubic supercell of length $16 \AA$ and sample the first $\mathrm{BZ}$ at the

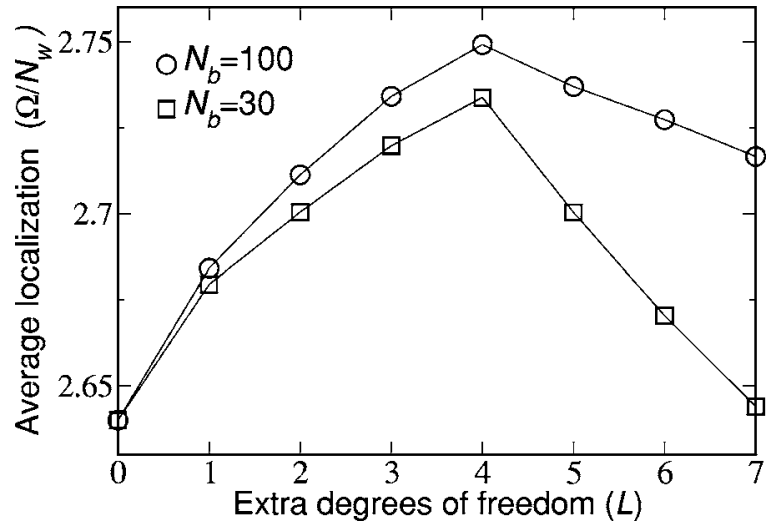

FIG. 4. Average spread of the WFs of the $\mathrm{Si}_{5}$ cluster for different values of $N_{b}$ and $L$.

$\Gamma$ point. To test the dependence on the size of the external localization space we consider the two cases $N_{b}=30$ and $N_{b}=100$. We set $M=10$ corresponding to the number of occupied states, and calculate the average localization per WF, $\langle\Omega\rangle=\Omega / N_{w}$, for $L=0, \ldots, 7$. The result is shown in Fig. 4. For $L=0$ there is no difference between the two cases since the WFs are constructed entirely from the occupied eigenstates. However, for $L \geqslant 0$ the larger space available for the extra degrees of freedom leads to an improved localization when $N_{b}=100$. Apart from this general improvement in localization, there is no qualitative difference between the WFs obtained with $N_{b}=30$ and $N_{b}=100$ for a given $L$. We note that both curves have a maximum for $L=4$, corresponding to a total of 14 WFs. This particular set of WFs together with their centers is shown in Fig. 3(b). The fact that this set of WFs respects the symmetry of the cluster is a special property of the $L=4$ solution: For other values of $L$, including $L=0$, the WFs break the symmetry of the $\mathrm{Si}_{5}$ cluster. This indicates that the solution corresponding to the maximal value of $\langle\Omega\rangle$ has a special meaning. Indeed, the value $N_{w}$ $=14$ could also have been anticipated from physical arguments. Starting from a set of four $s p^{3}$ orbitals located at each $\mathrm{Si}$ atom we expect bonding and antibonding states to form between pairs of aligned orbitals belonging to nearest neighbor pairs of $\mathrm{Si}$ atoms. These bonding states can be identified as the six bond-centered WFs shown to the far left in Fig. 3. The two "top" Si atoms have three nearest neighbors and thus a single $s p^{3}$ orbital is left as a lone pair (middle WF). The remaining three $\mathrm{Si}$ atoms each have two nearest neighbors and consequently two $s p^{3}$ orbitals are left as lone pairs (right-most WF). In total this adds up to 14 orbitals. The antibonding counterparts of the bonding states formed between nearest neighbors are not brought into play for $L=4$, because they are much less localized than the bonding states. However, by setting $L=10$ and thus searching for a total of $20 \mathrm{WFs}$, the antibonding states are picked out as EDF and we obtain a full set of $s p^{3}$ orbitals. This solution has, however, a smaller value for $\langle\Omega\rangle$ than the solution at $L=4$.

\section{B. Copper crystal}

To illustrate the method in the case of a periodic system we consider the construction of WFs for a copper crystal. 


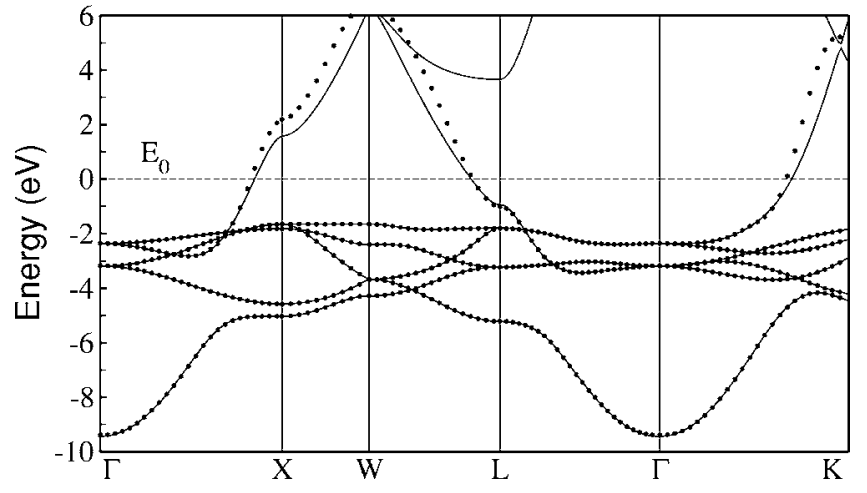

FIG. 5. Band structure of $\mathrm{Cu}(\mathrm{fcc})$. The full lines are the original DFT bands and the dots are the approximate bands computed from a set of six WFs $\left(N_{w}=6\right)$. The WFs have been constructed using $11 \times 11 \times 11 \mathbf{k}$ points and keeping all occupied states in the localization space, i.e., $E_{0}=0.0 \mathrm{eV}$ relative to the Fermi level.

This system was also studied by Souza et al. ${ }^{18}$ using their disentangling method to obtain the WFs. Our results are in close agreement with those obtained by Souza et al., and this indicates the similarity of the two methods for periodic systems.

We use the primitive fcc unit cell and sample the first BZ on a uniform $(11,11,11)$ Monckhorst pack grid containing the $\Gamma$ point. To obtain a minimal set of WFs describing the $\mathrm{Cu} d$ and $s$-bands we set $N_{w}=6$. We construct two sets of WFs corresponding to two different values of $E_{0}$ : (i) $E_{0}=0.0 \mathrm{eV}$ and (ii) $E_{0}=3.0 \mathrm{eV}$, relative to the Fermi level. In the first case the resulting WFs will span at least the occupied subspace and thus the electronic structure described by the WFs will be correct below $E_{F}$. In the second case the electronic structure will be correct up to $3 \mathrm{eV}$ above $E_{F}$, however, since this is a stronger restriction on the localization space we must expect that the resulting WFs will be less localized than those obtained in (i). In Figs. 5 and 6 we show the original DFT bands together with the approximate bands computed by diagonalizing the Hamiltonian within the subspace spanned by the WFs of cases (i) and (ii), respectively. In both cases we see a very good agreement between the exact and approximate bands below $E_{0}$. At higher energies the approximate bands deviate from the exact bands, indicating that the EDF which optimize the localization of the WFs do not co-

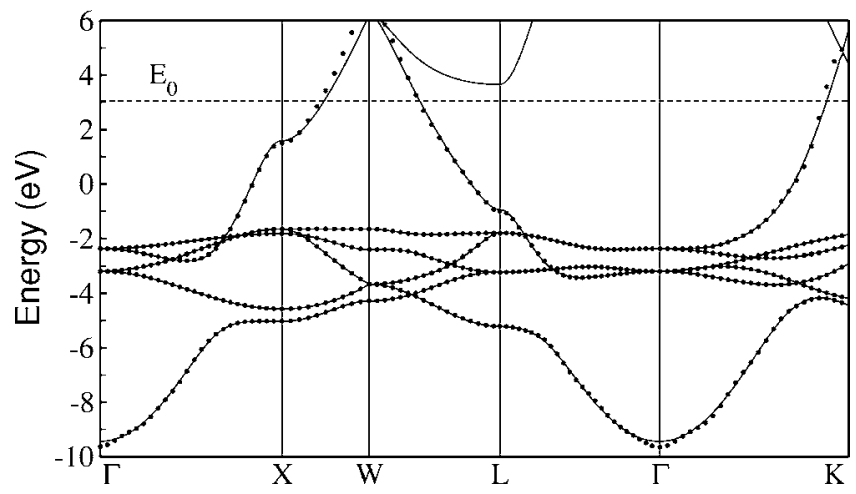

FIG. 6. Like Fig. 5 except that the WFs have been generated with $E_{0}=3.0 \mathrm{eV}$. (a)

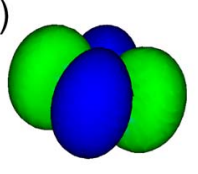

(b)

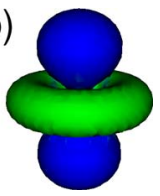

FIG. 7. (Color online) Two $d$-like WFs for $\mathrm{Cu}(\mathrm{fcc})$. The orbitals are centered at the atoms (not shown).

incide with specific Bloch eigenstates. The quality of the WF bands below $E_{0}$ depends on the number of $\mathbf{k}$ points used to construct the WFs. This is because the band diagram must be constructed starting from fully localized functions, which means that the coupling matrix elements must be truncated beyond a cut-off distance given approximately by $N_{i} / 2$ unit cells in the direction $\mathbf{a}_{i}$. Thus the repeated cell, or equivalently the number of $\mathbf{k}$ points, must be so large that the WFs have decayed sufficiently between the repeated images.

Both sets of WFs consist of five atom-centered $d$ orbitals and a single $s$-like orbital centered in one of the two interstitial sites. The $d$ orbitals are more or less identical for the two cases, and two examples are shown in Fig. 7. Contour plots of the $s$-like orbital is shown in Figs. 8(b) and 8(c) for cases (i) and (ii), respectively. The plots indicate that the $s$ orbital of case (ii) is less localized than the one obtained in case (i). That this is indeed correct follows from the value of the spread functional $\Omega$, which is higher for (i) than for (ii).

The minimal set of WFs obtained with $N_{w}=6$ breaks the symmetry of the fcc crystal because the $s$-like orbital is located in one of the interstitial sites leaving the other empty. As demonstrated by Souza et al. ${ }^{18}$ the symmetry can be restored by using seven WFs per primitive cell instead of six. In Fig. 9 we show the band structure obtained from a set of WFs generated with $N_{w}=7$ and $E_{0}=0.0 \mathrm{eV}$. We note that very high-energetic states are now selected as the optimal EDF. This solution can therefore only be obtained for rather (a)

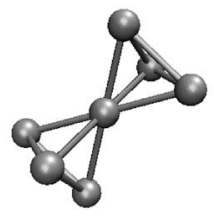

(c)

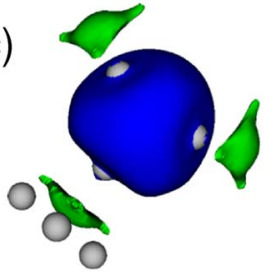

(b)

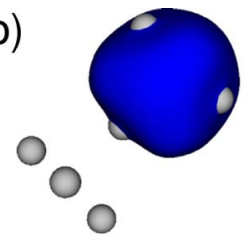

(d)

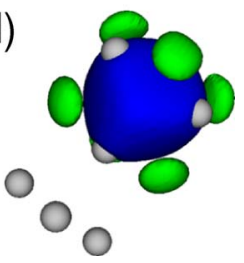

FIG. 8. (Color online) (a) Two tetrahedral interstitial sites in the fcc crystal. (b)-(d) Contour plots of the $s$-like WF obtained for $\mathrm{Cu}$ (fcc). The WFs in (b) and (c) have been generated with $N_{w}=6, E_{0}$ $=0.0 \mathrm{eV}$ and $N_{w}=6, E_{0}=3.0 \mathrm{eV}$, respectively. Both WFs are located in one of the interstitial sites. The WF in (d) corresponds to $N_{w}=7$ and $E_{0}=0.0 \mathrm{eV}$. In this case there is an equivalent WF located in the other interstitial site. The same contour value has been used for all plots. 


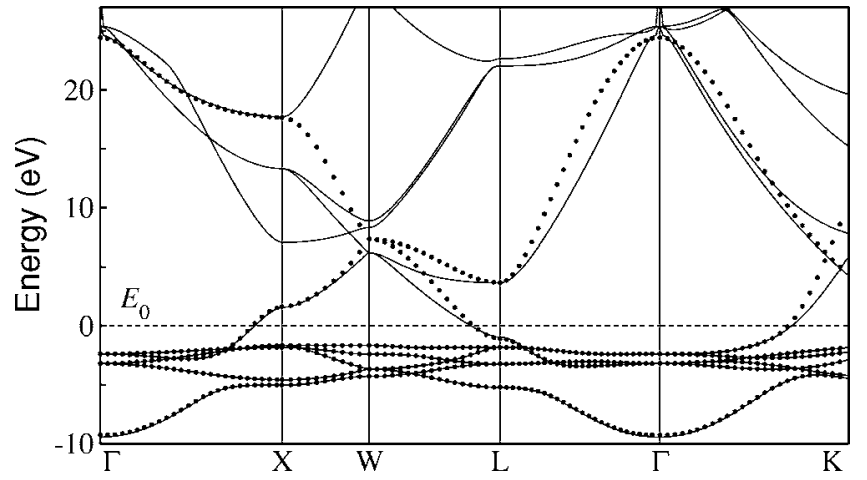

FIG. 9. Like Fig. 5 except that the WFs have been generated with $N_{w}=7$.

large external localization spaces, i.e., $N_{b} \geqslant 9$. The five $d$-like WFs are unchanged, but now we obtain two equivalent $s$-like WFs located in each of the two interstitial sites thereby restoring the fcc symmetry, see Fig. 8(d). We have calculated the average localization $\langle\Omega\rangle$ for $N_{w}=6,7,8$, and found that the maximum is attained for the symmetric solution with $N_{w}=7$.

\section{Nitrogen absorption on $\mathrm{Cu}(\mathbf{1 0 0})$}

In this section we study the WFs of a copper (100)surface covered with half a mono-layer of nitrogen atoms. As the system is neither periodic (in all directions) nor isolated, it represents a very general situation. The section is divided into two parts. In the first part the WFs are constructed and analyzed, and in the second part we use the obtained WFs to study the chemisorption of nitrogen within the NewnsAnderson model.

\section{Wannier function analysis}

We model the $\mathrm{Cu}(100)$ surface by a slab with a thickness of two atomic layers. The supercell contains four $\mathrm{Cu}$ atoms and a single $\mathrm{N}$ atom adsorbed in a hollow site, and its height is such that the surface slabs are separated by $9.0 \AA$ of vacuum. A topographic top view of the surface is shown in Fig. 10. We sample the first BZ on a uniform $(7,7,1)$ Mon-

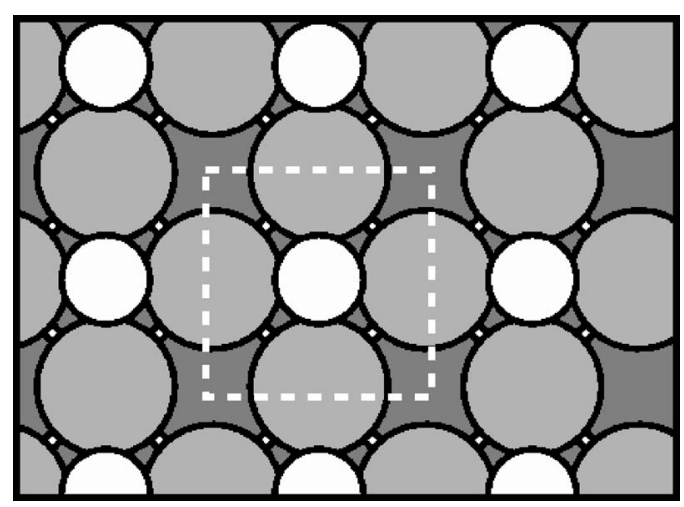

FIG. 10. Topographic view on the $\mathrm{Cu}(100)$ surface with adsorbed nitrogen. The white spheres are nitrogen, while the light gray spheres represent the $\mathrm{Cu}$ surface layer. A supercell is indicated.

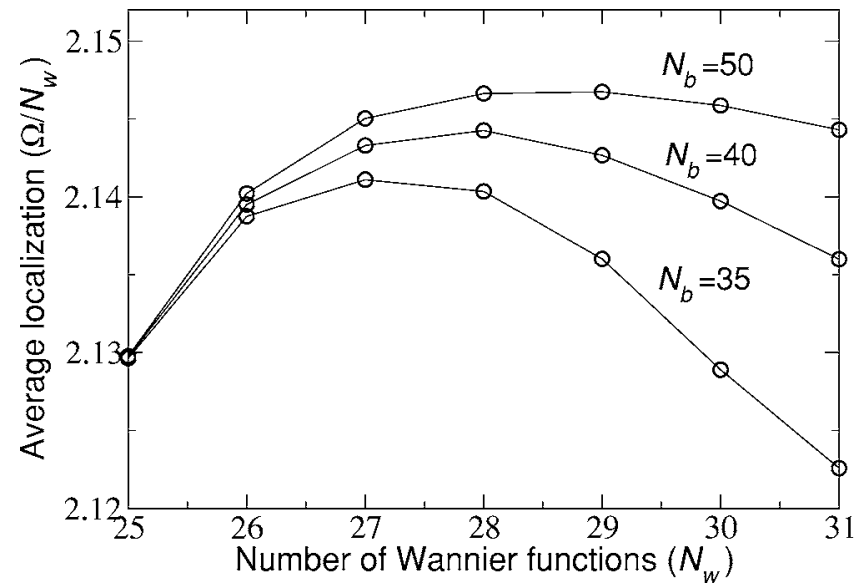

FIG. 11. Average localization of the WFs of the nitrogen covered $\mathrm{Cu}$ surface for different values of $N_{b}$ and $N_{w}$.

ckhorst pack grid containing the $\Gamma$ point.

Let us start by considering what we can expect to find on the basis of our previous experience. First, the result from the copper crystal suggests that a minimal description of the metal surface is obtained with five $d$ orbitals and a $s$-like orbital per $\mathrm{Cu}$ atom. Since there are four $\mathrm{Cu}$ atoms per supercell this gives a total of 24 WFs. Next, the similarity between the valency of $\mathrm{N}$ and $\mathrm{Si}$ together with our experience from the $\mathrm{Si}_{5}$ cluster points to a description of the nitrogen atom in terms of $s p^{x}$ hybrides.

In Fig. 11 we have plotted the average localization $\langle\Omega\rangle$ of the obtained WFs as a function of $N_{w}$ for three different sizes of the external localization space corresponding to $N_{b}=35$, 40,50 . In all cases we have set $E_{0}=E_{F}$ in order to ensure that the occupied eigenstates are exactly reproduced by the WFs. As expected, the localization improves as the size of the external localization space increases. In addition, the maximum of $\langle\Omega\rangle$ shifts toward larger $L$ values as $N_{b}$ is increased. Specifically the maximum shifts from $N_{w}=27$ to $N_{w}=29$ as $N_{b}$ is increased from 35 to 50 . This is not unexpected since we know that $\langle\Omega\rangle$ will be a monotonically increasing function of $L$ in the limit $N_{b} \rightarrow \infty$, see discussion in Sec. IV B. Again we stress that it is only the degree of localization of the WFs that change with $N_{b}$ for a fixed $L$, and not their qualitative form. Thus the chemical picture provided by the WFs does not change with $N_{b}$. In fact, for all the values of $N_{b}$ we obtain 20 highly localized $d$ orbitals (five located on each of the four $\mathrm{Cu}$ atoms) and four $s p^{3}$ orbitals centered on the $\mathrm{N}$ atom, see Fig. 13. The remaining $N_{w}-24 \mathrm{WFs}$ are the less localized $s$-like orbitals of $\mathrm{Cu}$. Thus, as $N_{w}$ is increased beyond 24 , the number of $s$-like $\mathrm{Cu}$ WFs simply increases correspondingly.

To gain further insight into the dependence of the WFs on $N_{b}$ and $N_{w}$, we show in Fig. 12 the average localization of the $d, s p^{3}$, and $s$ orbitals, separately. It is clear that the $N_{b}$ dependence as well as the maximum of $\langle\Omega\rangle$ are almost exclusively related to the $\mathrm{Cu} s$ orbitals. Except for the case $N_{b}$ $=50$, which is in fact somewhat extreme since states of 20 $\mathrm{eV}$ above the Fermi level are included in the external localization space, the average spread of the $\mathrm{Cu} s$ orbitals is maximal for $N_{w}=28$. This corresponds to one $s$ orbital per $\mathrm{Cu}$ 


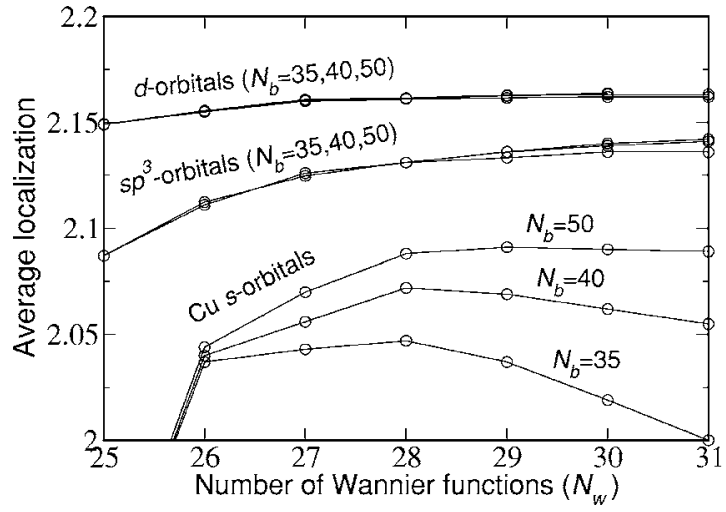

FIG. 12. Average localization of the $d$-, $s p^{3}$-, and $s$-like WFs considered separately for different values of $N_{b}$ and $N_{w}$.

which is exactly what we anticipated from the analysis of the copper crystal.

We end by summarizing the chemical picture obtained from the WF analysis: For $N_{w}=28$ the $\mathrm{Cu}$ surface is described by the minimal set of WFs consisting of five $d$ - and one $s$-like orbital per atom. For the nitrogen we obtain four $s p^{3}$ hybrids oriented as indicated in Fig. 13.

\section{Adsorption in the Newns-Anderson model}

The WFs can be used to obtain a detailed and consistent picture of the hybridization occurring between the nitrogen states and the states of the substrate. As we shall see the analysis gives a complete account for the shape of the projected density of states of a given $\mathrm{N}$ orbital, in terms of the bare orbital energy, a coupling strength, and the density of states of the so-called group orbital.

In the Newns-Anderson model, ${ }^{23}$ one considers an adsorbate state, $|a\rangle$, of energy $\varepsilon_{a}=\langle a|H| a\rangle$, coupled to a continuum of states, $|k\rangle$, representing the substrate. The coupling matrix
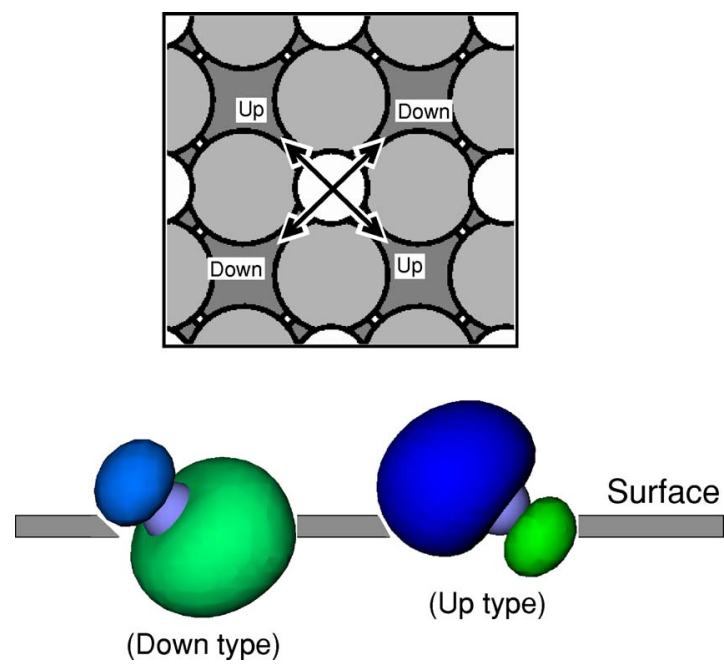

FIG. 13. (Color online) Orientation of the four $s p^{3}$-like WFs belonging to the $\mathrm{N}$ atom. Seen from above the orbitals point to the bridge sites of the $\mathrm{Cu}$ atoms in the surface. Each orbital points either up from or down into the surface. An example of each type is shown in the lower panel. elements are denoted by $V_{k}=\langle a|H| k\rangle$. A particularly useful formulation can be obtained by introducing the normalized group orbital, $|g\rangle=V^{-1} \Sigma_{k} V_{k}|k\rangle$, where $V=\left(\Sigma_{k}\left|V_{k}\right|^{2}\right)^{-1 / 2}$. It is easily checked, that the coupling between $|a\rangle$ and any substrate state orthogonal to $|g\rangle$ vanishes. Consequently $|a\rangle$ is coupled to the substrate via the group orbital only, and the coupling is given by $V$, i.e., $V=\langle a|H| g\rangle$.

Physical quantities such as the projected density of states (PDOS) of the adsorbate state and the hybridization part of the adsorption energy, can be obtained from the retarded adsorbate Green's function, which in turn follows from the three quantities $\varepsilon_{a}, V$ and $\rho_{g}^{0}(\varepsilon)$, where $\rho_{g}^{0}$ denotes the PDOS of the group orbital in the absence of coupling to the adsorbate state. Often $\rho_{g}^{0}(\varepsilon)$ is referred to as the band to which the adsorbate is coupled.

The $s p^{3} \mathrm{WFs}$ of the $\mathrm{N}$ atom are not well suited as a starting point for applications of the Newns-Anderson model, since they do not represent the energy levels of the free atom. This problem can be overcome by diagonalizing the Hamiltonian matrix in the WF basis, within the subspace spanned by the four $s p^{3}$ orbitals. The result of the subspace diagonalization is a set of four atomic orbitals consisting of one $s$-like and three $p$-like orbitals, each centered at the $\mathrm{N}$ atom. Two of the $p$ orbitals lie in the surface plane (the $x y$ plane) and are directed along the arrows shown in Fig. 13, while the third is oriented along the surface normal (the $z$ axis). We shall refer to the $p$ orbitals as $p_{x}, p_{y}$, and $p_{z}$, respectively. The energies corresponding to the atomic orbitals are (in electronvolts): $\varepsilon_{s}=-14.8, \varepsilon_{z}=-2.4, \varepsilon_{x}=-3.7$, and $\varepsilon_{y}=-4.2$. We notice, that the energy of the $p_{x}$ and $p_{y}$ orbitals differ even though the symmetry of the system suggests that they should be equal. The reason for this is that the WFs break the fourfold rotation symmetry of the system, i.e., the subspace spanned by the four $s p^{3}$ WFs is not invariant under the same symmetry transformations as the Hamiltonian. This is not surprising, since the WFs are constructed solely from a criterion of maximal localization and no attempts are made to conserve symmetries. On the other hand we have found that by increasing the parameter $E_{0}$ above the value $E_{0}=E_{F}$ used in the present example, the symmetry between $p_{x}$ and $p_{y}$ can be restored. The price one has to pay is that the copper $s$-like WFs become less localized due to the further constrains on the localization space implied by the larger value of $E_{0}$. From the Hamiltonian in the WF basis we can also obtain the coupling $V$ between each of the atomic nitrogen orbitals and its corresponding group orbital. These are quite similar and vary from 3.1 to $3.8 \mathrm{eV}$.

In Fig. 14 we show the calculated PDOS for each of the three nitrogen $p$ orbitals (upper panel). Although the on-site energies of the $p_{x}$ and $p_{y}$ orbitals differ (as discussed above), their PDOS are rather similar. The PDOS of the corresponding group orbitals have been calculated with all coupling matrix elements to the $\mathrm{N}$ orbitals set to zero, i.e., the adsorbate states have effectively been decoupled from the surface. The result is shown in the lower panel of Fig. 14. For all three orbitals, the on-site energies lie within the band. Due to the strong coupling, bonding, and antibonding resonances are formed at the band edges around -7 and $0 \mathrm{eV}$ as can be seen in the upper panel of the figure. This is the limit of strong 


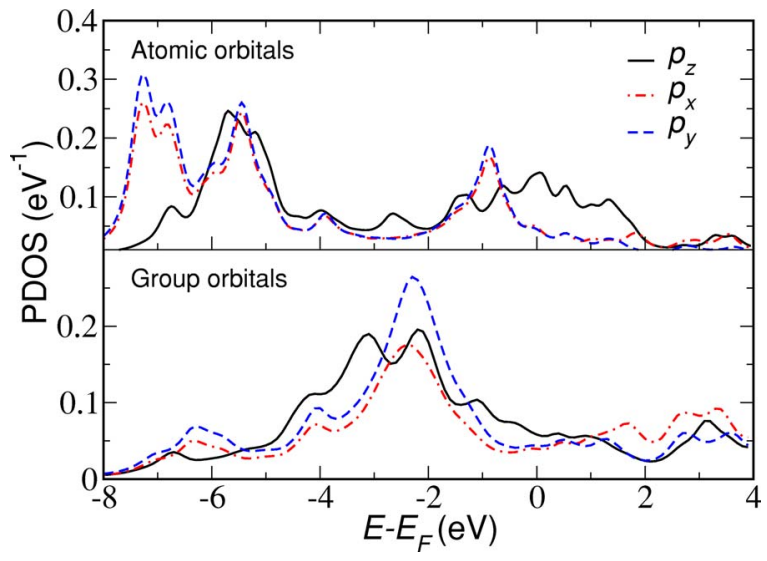

FIG. 14. (Color online) Top: PDOS for the atomic $p$ orbitals obtained by diagonalizing the Hamiltonian in the subspace spanned by the $s p^{3}$ WFs of the $\mathrm{N}$ atom. Bottom: PDOS for the group orbitals corresponding to each of the atomic $p$ orbitals.

chemisorption. ${ }^{23}$ Since the four orbitals span all states with significant weight on the $\mathrm{N}$ atom, this representation provides a full representation of the nitrogen bonding.

\section{CONCLUSIONS}

We have presented a practical method for constructing partly occupied WFs for a wide range of systems. The method employs a bonding-antibonding closing procedure to filter out a set of unoccupied states, called the extra degrees of freedom, which serve to improve the localization of the WFs. The determination of the extra degrees of freedom is based on a minimization of the spread of the resulting WFs. We derived expressions for the gradients of the spread functional and showed how these can be combined with a Lagrange multiplier scheme to minimize the spread functional.

The generality of the scheme was demonstrated by applying the method to three different systems. As an example of an isolated system, we considered a $\mathrm{Si}_{5}$ cluster, and showed how different sets of WFs could be obtained by varying the number of extra degrees of freedom. A similar analysis was performed for a copper crystal, where we found results very similar to those of Souza et al. ${ }^{18}$ Finally we studied in detail the WFs of a $\mathrm{Cu}(100)$ surface with a nitrogen coverage of 0.5 . In many cases we were able to obtain a special set of WFs with a particularly high degree of symmetry and localization, by maximizing the average spread of the WFs. Moreover, the condition of maximal average localization was shown to coincide with a complete matching of bonding and antibonding states.

\section{ACKNOWLEDGMENTS}

We acknowledge support from the Danish Center for Scientific Computing through Grant No. HDW-1101-05.

\section{APPENDIX: SPREAD FUNCTIONAL FOR VANDERBILT ULTRASOFT PSEUDOPOTENTIALS}

For Vanderbilt ultra-soft pseudopotentials ${ }^{21}$ the optimal smoothness of the pseudo-wave functions is obtained by re- laxing the norm-conserving constrains for the pseudo-wave functions. This results in a generalized orthonormality relation $^{21}$

$$
\left\langle\psi_{i}|S| \psi_{j}\right\rangle=\delta_{i j}
$$

The Hermitian operator $S$ is given by

$$
S=1+\sum_{I} \sum_{n m} q_{n m}\left|\beta_{n}^{I}\right\rangle\left\langle\beta_{m}^{I}\right|
$$

where the index $I$ denotes the atoms in the system, and $q_{n m}$ is given by

$$
q_{n m}=\int d \mathbf{r} Q_{n m}^{I}(\mathbf{r}) .
$$

The functions $\left\{\beta_{n}^{I}\right\}$ and $\left\{Q_{n m}^{I}\right\}$ are all localized functions centered at atom $I$. The functions $\left\{Q_{n m}^{I}\right\}$ describe the augmentation charge not contained in the smooth pseudowave functions, and they must therefore be included in the calculation of the spread of the wave functions.

\section{Large supercells}

In the case of large supercells, using the $\Gamma$-point approximation, Bernasconi and Madden ${ }^{24}$ derived the following expression for the contribution to $Z_{\alpha}^{(0)}$ from the augmentation charges $Q_{n m}^{I}(\mathbf{r})$ :

$$
Z_{\alpha, i j}^{(u s, 0)}=\sum_{I, n m}\left\langle\psi_{i} \mid \beta_{m}^{I}\right\rangle\left\langle\beta_{n}^{I} \mid \psi_{j}\right\rangle \int d \mathbf{r} e^{-\mathrm{i} \mathbf{G}_{\alpha} \cdot \mathbf{r}} Q_{m n}(\mathbf{r}) .
$$

\section{Periodic systems}

For the periodic case, using a uniform k-point grid, we write $Z_{\alpha}^{u s}$ as

$$
Z_{\alpha}^{u s}=\sum_{\mathbf{k k}^{\prime}} Z_{\alpha}^{(u s) \mathbf{k} \mathbf{k}^{\prime}} .
$$

Here again we use the exact correspondence between the Bloch states $\psi_{n \mathbf{k}}$ and the $\Gamma$-point eigenstates of the repeated cell. In the repeated cell we use the notation

$$
h_{i \mathbf{k}}^{I n \mathbf{t}}=\left\langle\psi_{i \mathbf{k}} \mid \beta_{n}^{I, \mathbf{t}}\right\rangle=\left\langle\psi_{i \mathbf{k}} \mid \beta_{n}^{I, \mathbf{t}=\mathbf{0}}\right\rangle e^{i \mathbf{k} \cdot \mathbf{R}_{\mathrm{t}}}
$$

and

$$
Q_{n m}^{I \mathbf{t}}=\sum_{\mathbf{G}} Q_{n m}(\mathbf{G}) e^{-i \mathbf{G} \cdot\left(\mathbf{r}-\mathbf{R}_{\mathbf{t}}\right)} .
$$

$\mathbf{R}_{\mathbf{t}}$ is here a real space translation vector, given in terms of the basis $\mathbf{a}, t_{1} \mathbf{a}_{1}+t_{2} \mathbf{a}_{2}+t_{3} \mathbf{a}_{3}, \mathbf{t}=\left(t_{1}, t_{2}, t_{3}\right)$. We will use $h^{I n}$ $=h^{I, \mathbf{t}=\mathbf{0}, n}$ in what follows. Inserting $h_{i \mathbf{k}}^{I n \mathbf{t}}$ and $Q_{n m}^{I \mathbf{t}}$ from Eqs. (A6) and (A7), together with the Bloch states $\psi_{i \mathbf{k}}$ into the $\Gamma$-point expression for $Z_{\alpha}^{(u s, 0)}$ in Eq. (A4), we find

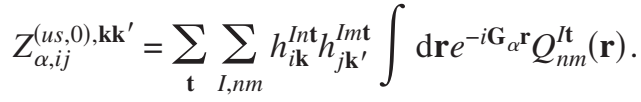

The sum over $\mathbf{t}$ is for $t_{i}=0, \ldots, N_{i}-1$, see Eq. (21). Inserting the left-hand side of Eqs. (A6) and (A7), and rearranging, we find 


$$
\begin{aligned}
Z_{\alpha, i j}^{(u s, 0), \mathbf{k} \mathbf{k}^{\prime}}= & \sum_{I, n m} h_{i \mathbf{k}}^{I n} h_{j \mathbf{k}^{\prime}}^{I m} \sum_{\mathrm{t}} e^{-i\left(\mathbf{k}-\mathbf{k}^{\prime}\right) \mathbf{R}_{\mathrm{t}}} \\
& \times \sum_{\mathbf{G}} Q_{n m}(\mathbf{G}) e^{-i \mathbf{G} \cdot \mathbf{R}_{\mathrm{t}}} \int \mathrm{d} \mathbf{r} e^{i\left(\mathbf{G}-\mathbf{G}_{\alpha}\right) \mathbf{r}} .
\end{aligned}
$$

Finally, we arrive at our expression for $Z_{\alpha, i j}^{(u s, 0), \mathbf{k k}^{\prime}}$

$$
Z_{\alpha, i j}^{(u s, 0), \mathbf{k k}^{\prime}}=\sum_{I, n m} h_{i \mathbf{k}}^{I n} h_{j \mathbf{k}^{\prime}}^{I m} \sum_{\mathbf{t}} e^{-i\left(\mathbf{k}-\mathbf{k}^{\prime}-\mathbf{G}_{\alpha}\right) \mathbf{R}_{\mathrm{t}}} Q_{n m}\left(\mathbf{G}=\mathbf{G}_{\alpha}\right),
$$

which is nonzero only when $\mathbf{k}$ and $\mathbf{k}^{\prime}$ fulfills the condition in Eq. (29). Again we see that this expression contains the $\Gamma$-point formalism, Eq. (A4), as a special case.
${ }^{1}$ G. H. Wannier Phys. Rev. 52, 191 (1937).

${ }^{2}$ N. W. Ashcroft and N. D. Mermin, Solid State Physics (Saunders, New York, 1976).

${ }^{3}$ G. D. Mahan, Many-Particle Physics (Plenum Press, New York, 1990).

${ }^{4}$ R. Resta, Rev. Mod. Phys. 66, 899 (1994).

${ }^{5}$ R. D. King-Smith and D. Vanderbilt, Phys. Rev. B 47, R1651 (1993).

${ }^{6}$ S. Goedecker, Rev. Mod. Phys. 71, 1085 (1999); C. K. Skylaris, A. A. Mostofi, P. D. Haynes, O. Dieguez, and M. C. Payne, Phys. Rev. B 66, 035119 (2002); D. R. Bowler, T. Miyazaki and M. J. Gillan, J. Phys.: Condens. Matter 14, 2781 (2002); J. L. Fattebert and J. Bernholc, Phys. Rev. B 62, 1713 (2000); J. Kim, F. Mauri and G. Galli, ibid. 52, 1640 (1995).

${ }^{7}$ A. Calzolari, N. Marzari, I. Souza, and M. B. Nardelli, Phys. Rev. B 69, 035108 (2004).

${ }^{8}$ K. S. Thygesen and K. W. Jacobsen, Chem. Phys. (to be published) cond-mat/0501238.

${ }^{9}$ S. F. Boys, Rev. Mod. Phys. 32, 296 (1960).

${ }^{10}$ J. M. Foster and S. F. Boys, Rev. Mod. Phys. 32, 300 (1960).

${ }^{11}$ C. Edmiston and K. Ruedenberg, Rev. Mod. Phys. 35, 457 (1963).
${ }^{12}$ J. Pipek and P. G. Mezey, J. Chem. Phys. 90, 4916 (1989)

${ }^{13}$ G. Berghold, C. J. Mundy, A. H. Romero, J. Hutter, and M. Parrinello, Phys. Rev. B 61, 10040 (2000).

${ }^{14}$ P. L. Silvestrelli, Phys. Rev. B 59, 9703 (1999).

${ }^{15}$ N. Marzari and D. Vanderbilt, Phys. Rev. B 56, 12847 (1997).

${ }^{16}$ M. Iannuzzi and M. Parrinello, Phys. Rev. B 66, 155209 (2002).

${ }^{17}$ K. S. Thygesen, L. B. Hansen, and K. W. Jacobsen, Phys. Rev. Lett. 94, 026405 (2005).

${ }^{18}$ I. Souza, N. Marzari, and D. Vanderbilt, Phys. Rev. B 65, 035109 (2001).

${ }^{19}$ R. Resta and S. Sorella, Phys. Rev. Lett. 82, 370 (1999).

${ }^{20}$ B. Hammer, L. B. Hansen, and J. K. Nørskov, Phys. Rev. B 59, 7413 (1999); S. R. Bahn and K. W. Jacobsen, Comput. Sci. Eng. 4, 56 (2002); The Dacapo code can be downloaded at http:// www.camp.dtu.dk/campos

${ }^{21}$ D. Vanderbilt, Phys. Rev. B 41, R7892 (1990).

${ }^{22}$ K. Raghavachari and V. Logovinsky, Phys. Rev. Lett. 55, 2853 (1985).

${ }^{23}$ D. M. Newns, Phys. Rev. 178, 1123 (1969).

${ }^{24}$ L. Bernasconi and P. A. Madden, J. Mol. Struct.: THEOCHEM 544, 49 (2001). 